\title{
The Fuzzy Model for Diagnosis of Animal Disease
}

\author{
Xiao Jianhua, Shi Luyi, Zhang Yu, Gao Li, Fan Honggang, \\ Ma Haikun, and Wang Hongbin* \\ College of Veterinary Medicine, NorthEast Agricultural University, \\ Harbin, Heilongjiang Province, P.R. China \\ Voice: +86-451-55190470 \\ 66229894 @qq. com
}

\begin{abstract}
The knowledge of animal disease diagnosis was fuzzy; the fuzzy model can imitate the character of clinical diagnosis for veterinary. The fuzzy model of disease, the methods for class the disease group of differential diagnosis and the fuzzy diagnosis model were discussed in this paper.
\end{abstract}

Keywords: disease, fuzzy, diagnosis, model.

\section{Introduction}

Diagnosis knowledge of animal disease is fuzzy, and the establishment of representation and reasoning model for animal diagnosis knowledge based on fuzzy theory can reflects the nature of medical diagnosis to some extent.

It was reported that the fuzzy inference model for diagnosis of bone tumors based on fuzzy set theory and medical statistics knowledge can not only simulate the diagnosis of bone tumors, but also can adjust the parameters to improve the accuracy of diagnosis with the accumulation of cases(Huikang Zhang, 2003).

In addition, the mathematical model for diagnosis of gastric cancer based on Fuzzy clustering and stepwise discriminate analysis method(Xiaoyan Lu,2003), the expert system for diagnosis of liver disease based on fuzzy reasoning(Carvalho V,2005) heart disease classification system (Shiomi S,1995) Dairy claudicating Forecast Expert System based on fuzzy logic(Pop HF,2001) etc.

\section{Materials and Methods}

\subsection{Knowledge Is Key in Government and Governance}

The symptoms, medical history, signs, laboratory data, pathology tests and other information of animal can be used as evidence of diagnosis. $X$ is the universe composed with all evidence in diagnosis of animal diseases, $x$ is one of evidence, different evidence with different effect which can be expressed as ambiguity when diagnosis of

\footnotetext{
* Corresponding author.
} 
diseases. Therefore the fuzzy mathematical model can be build for disease diagnosis, namely:

For any evidence of $x \in X$, the mappings: $x \mid \rightarrow \mu_{\sim}(x) \in[0,1]$ exist.

The above mappings can be regard as the relationship between $x$ and one disease. The evidence collection of one disease can be established through this Mapping

$$
\underset{\sim}{A}=\left\{\left(x \mid \mu_{\sim}(x)\right)\right\}, \forall x \in X
$$

$\underset{\sim}{A}$ is the collection of evidence for diagnosis of one disease, which may include the symptoms, history and epidemiological data, $\underset{\sim}{A}$ is the subset of $X, \mathrm{~F}(\mathrm{X})$ is all collection in $X$. Then $\underset{\sim}{A} \subset F(X) . \mu_{A}(x)$ is the membership function of elements $x$ in disease $\mathrm{A}$, for a specific $x, \mu_{A}(x)$ is Degree of membership of $x$ for $\underset{\sim}{A}$, is extent of the role in the course of the disease diagnosis in another words.

Supposing:

$\mathrm{A}=$ Rumen indigestion

$x_{1}=$ The potable water is insufficient after crossing the food.

$$
\begin{aligned}
& \mu_{\dot{\sim}\left(x_{1}\right)}=0.1 \\
& \mu_{\sim}\left(x_{2)}=0.25\right. \\
& \mu_{\sim}\left(x_{3)}=0.2\right. \\
& \mu_{\sim}\left(x_{4)}=0.8\right. \\
& \mu_{\sim}\left(x_{5)}=0.6\right.
\end{aligned}
$$

$x_{2}=$ Has eaten feed which easily to swell

$x_{3}=$ Change feed suddenly

$x_{4}=$ The rumen contents was hardly, assumes the pasta type

$x_{5}=$ Left side of girth of paunch expands obviously

$x_{6}=$ Rumen discharge gas when puncture upside rumen, and discharge digestion food when puncture lower part

$x_{7}=$ Indentation appeared in skin when palpate rumen

$$
\begin{aligned}
& \mu_{\sim}\left(x_{6}\right)=0.7 \\
& \mu_{\dot{\sim}}\left(x_{7}\right)=0.8
\end{aligned}
$$

The collection of element for diagnose rumen indigestion can be express as:

$$
\underset{\sim}{A}=\frac{0.1}{x_{1}}+\frac{0.25}{x_{2}}+\frac{0.2}{x_{3}}+\frac{0.8}{x_{4}}+\frac{0.6}{x_{5}}+\frac{0.7}{x_{6}}+\frac{0.8}{x_{7}}
$$

\subsection{The Division of Differential Diagnosis Disease Group}

Similarity or Relevance may exist between one disease and another disease. In order to diagnose, the relationships between diseases must be find and express in some forms. Diseases of one kind of animal often have as many as hundreds of species, it is impossible to express hundreds of the relationship between theses diseases, in fact, there is no need for such a gesture. Between each kind of disease has the relations by no means, it is easy to distinguish among diseases with few relations. The key of diagnosis is to differentiate diseases that exist relation. Therefore hundreds kind of diseases must be divide into many differential diagnosis group at first. Diseases that have big similarity must be division is one group.

The relations need to be established among theses diseases which belong to one differential diagnosis group. It is helpful to discover similar diseases that to established this kind of relations. The similarity among diseases is fuzzy, and can be express in degree of membership. And the relations among diseases may express for the following fuzzy relationship. 
Supposes $A$ one group of diseases that need to be differential diagnose, $A=\left\{x_{1}, x_{2}, \cdots, x_{n}\right\}, x_{i}$ is the $i_{t h}$ disease.

$$
\underset{\sim}{R}=\left(\begin{array}{ccc}
r_{11} & \cdots & r_{1 n} \\
\vdots & \ddots & \vdots \\
r_{n 1} & \cdots & r_{n n}
\end{array}\right) \text { or } \underset{\sim}{R}=\left(r_{i j}\right)_{n \times n}
$$

And $r_{i j}=\underset{\sim}{R}\left(x_{i}, x_{j}\right)$. There was relationship between $i_{t h}$ disease and $j_{t h}$ disease.

By this model, diseases was divided into many groups, when diagnosis, the conclusion can be discover according to the similarity among diseases.

The differential diagnosis group may determined use the fuzzy distance law, Supposes $\mathrm{D}$ is a collection that need to be differential diagnose, $\mathrm{D}=\left\{D_{1}, D_{2}, \cdots, D_{m}\right\}$, $S$ is the collection composed of symptoms, $S=\left\{s_{1}, s_{2}, \cdots, s_{n}\right\}$. The distance between $D_{x}$ and $D_{y}$ can be express as:

$$
d\left(\underset{\sim}{D_{x}}, \underset{\sim}{D_{y}}\right)=\frac{1}{n} \sum_{i=1}^{n} W\left(s_{i}\right)\left|\underset{\sim}{D_{x}}\left(s_{i}\right)-\underset{\sim}{D_{y}}\left(s_{i}\right)\right|
$$

$d$ is the distance between $D_{x}$ and $D_{y}$, If one threshold value $\lambda$ is determined, when the distance between two diseases $d<\lambda$, then those two diseases belong to one differential diagnosis group.

\subsection{Animal Disease Fuzzy Diagnosis Mathematical Model}

The fuzzy model was established between disease and symptoms at begin, supposed $D$ is the collection that need to be differential diagnosed, $D=\left\{d_{1}, d_{2}, \cdots, d_{m}\right\}, S$ is the collection of symptoms $S=\left\{s_{1}, s_{2}, \cdots, s_{n}\right\}$. The fuzzy relationship between diseases and symptoms can be express as fuzzy matrix of $\mathrm{n}$ row and $\mathrm{m}$ list:

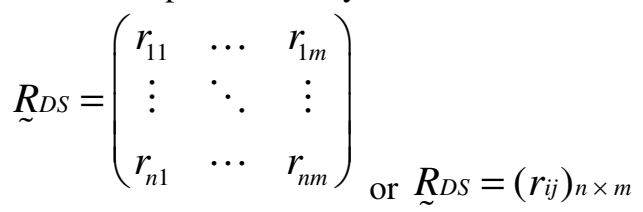

$\underset{\sim}{R_{D S}}$ is the fuzzy relationship between disease and symptom, $r_{i j}=\underset{\sim}{R}\left(x_{i}, y_{j}\right), 0<r_{i j}<1$ 。

The fuzzy distance model of diagnosis can be established based on the above model. Supposes D is the collection of diseases that need to be diagnosed, $\mathrm{D}=\left\{D_{1}, D_{2}, \cdots, D_{m}\right\}, S$ is the collection of symptoms, $S=\left\{s_{1}, s_{2}, \cdots, s_{n}\right\}$. Then the distance between disease $D_{x}$ and $D_{r}$ can be express as: 


$$
\begin{aligned}
& \left(d_{1}, d_{2}, \cdots, d_{m}\right)=\left(s_{1}, s_{2}, \cdots, s_{n}\right) \circ\left(\begin{array}{lll}
r_{11} & \cdots r_{1 j} \cdots & r_{1 m} \\
\vdots & & \vdots \\
r_{i 1} & \cdots r_{i j} \cdots & r_{i m} \\
\vdots & & \vdots \\
r_{n 1} & \cdots r_{n j} \cdots & r_{n m}
\end{array}\right) \\
& d_{j}\left(\underset{\sim}{D_{x}}, \underset{\sim}{D_{r}}\right)=\frac{1}{n} \sum_{i=1}^{n} W\left(s_{i}\right)\left|\underset{\sim}{D_{x}}\left(s_{i}\right)-\underset{\sim}{D_{r}}\left(s_{i}\right)\right| \quad \underset{\sim}{D_{r}}\left(s_{i}\right)=r_{i j}
\end{aligned}
$$

$\mathrm{d}=\left\{d_{1}, d_{2}, \cdots, d_{m}\right\}$, the most impossible disease is $\min \left\{d_{1}, d_{2}, \cdots, d_{m}\right\}$. the disease with most impossible can be determined by single factor fuzzy diagnosis model when there exist two diseases need to be diagnosed, however multi-factors fuzzy diagnosis model need to be established when many diseases need to be diagnosed, suppose D is one group disease need to be differential diagnosed, $\mathrm{D}=\left\{\underset{\sim}{D_{1}}, \underset{\sim}{D_{2}}, \cdots, \underset{\sim}{D_{m}}\right\}$, suppose $D_{x}$ is the disease need to be diagnosed. $\sigma$ is matching between treating disease and any disease in $\mathrm{D}$, suppose:

$$
\begin{gathered}
\sigma\left(\underset{\sim}{D_{i}}, \underset{\sim}{D_{x}}\right)=\max \left\{\sigma\left(\underset{\sim}{D_{k}}, \underset{\sim}{D_{x}}\right) \mid k=1,2, \cdots, m\right\} \\
\sigma\left(\underset{\sim}{D_{i}}, \underset{\sim}{D_{x}}\right)=1-\frac{1}{n} \sum_{j=1}^{n} W\left(s_{j}\right)\left|\underset{\sim}{D_{x}}\left(s_{j}\right)-{\underset{\sim}{D}}_{i}\left(s_{j}\right)\right| \\
i=\{1,2, \cdots, m\}, \quad j=\{1,2, \cdots, n\}
\end{gathered}
$$

It is most close that $D_{x}$ and $\underset{\sim}{D_{i}}$, then $\underset{\sim}{D_{i}}$ is determined the conclusion.

\subsection{Conclusion}

There are hundreds of diseases for animals, moreover the specialization degree is relatively low in veterinary, any veterinarian have to solve diseases of every kind of animal, and can solve internal medicine sickness, surgical sickness, the obstetrical disease, parasitic disease, the infectious disease and so on. Therefore, the expert system for animal disease must conform to the objective law of veterinarian clinical. The animal disease should be divides into some big group such as internal medicine sickness, surgical sickness, and then divide into many differential diagnosis groups. By different character of disease, the different knowledge expression and the inference method was developed. The fuzzy model in this paper has been used in some diseases, but it is necessary to apply in more diseases.

\section{Acknowledgements}

The authors thank the financial support from National Project of Scientific and Technical Supporting of China during the 11th Five-year Plan 2006BAD10A02-04. 


\section{References}

Carvalho, V., Naas, I., Mollo, M., et al.: Prediction of the Occurrence of Lameness in Dairy Cows using a Fuzzy-Logic Based Expert System-Part I. Agricultural Engineering International: the CIGR Ejournal VII, Manuscript IT 05 002, June 1-12 (2005)

Zhang, H., Qian, Z., Qu, J., et al.: Fuzzy reasoning model for bone tumor auxiliary diagnoses expert system. Journal of Forth Military Medical University 24(2), 182-186 (2003)

Shiomi, S., Kuroki, T., Jomura, H., et al.: Diagnosis of chronic liver disease from liver scintiscans by fuzzy reasoning. J. Nucl. Med. 36(4), 593-598 (1995)

Pop, H.F., Pop, T.L., Sarbu, C.: Assessment of heart disease using fuzzy classification techniques. Scientific World Journal 17(1), 369-390 (2001)

Lu, X., Guo, J.: The auxiliary diagnostic model for medicine mathematics based on fuzzy and gradually analysis. Journal of shanxi medical college 34(6), 499-502 (2003) 